\title{
Effect of Gastrodia elata-notoginseng decoction on blood pressure variability and inflammatory factors in old hypertensive patients with yin-deficiency and yang-hyperactivity syndrome.
}

\author{
Wang Dengqin ${ }^{1}$, Zhang Xiaoxue ${ }^{2}$, Song Guohong ${ }^{3}$, Li Lei ${ }^{4}$, Sun Bing ${ }^{5^{*}}$ \\ ${ }^{1}$ Clinical Medical Skills Center, Jining Medical University, PR China \\ ${ }^{2}$ Department of Neurology, Jining City Hospital of Traditional Chinese Medicine, PR China \\ ${ }^{3}$ Teaching-Research Office of Surgery, Jining Medical University, PR China \\ ${ }^{4}$ Teaching-Research Office of Diagnostics, Jining Medical University, PR China \\ ${ }^{5}$ Teaching-Research Office of Traditional Chinese Medicine, Jining Medical University, PR China
}

\begin{abstract}
Objective: This research was aimed to explore the decoction ability of Gastrodia elata-notoginseng of blood pressure variability and inflammatory factors in old hypertensive patients with yin-deficiency and yang-hyperactivity syndrome.

Methods: Totally 80 hypertensive patients with yin-deficiency and yang-hyperactivity syndrome were randomly divided into control group (40 cases) and treatment group (40 cases). Two groups received Lamlodipine $2.5 \mathrm{mg}$ q.d., one tablet daily), and the treatment group additionally received Gastrodia elatanotoginseng decoction. Both groups were continuously treated for 12 weeks.

Results: The treatment efficacy in treatment group $(\mathbf{8 5 . 0 \%})$ was higher than control group $(62.5 \%)$ and the difference was statistically significant $(\mathbf{P}<0.01)$. After treatment, various parameters of treatment group were significantly lower $(\mathrm{P}<0.01$ or $\mathrm{P}<0.05)$ than that of control group, including $24 \mathrm{~h}$ systolic pressure standard deviation ( $24 \mathrm{~h} \mathrm{SSD}$ ), $24 \mathrm{~h}$ diastolic pressure standard deviation ( $24 \mathrm{~h}$ DSD), diurnal systolic pressure Standard Deviation (dSSD), nocturnal systolic pressure standard deviation (nSSD), 24 h mean systolic blood pressure ( $24 \mathrm{~h}$ SBP), serum resistin level, C-reactive protein (CRP) level and adiponectin level.

Conclusions: Compared with Western medicine therapy only, Gastrodia elata-notoginseng decoction can further decrease blood pressure.
\end{abstract}

Keywords: Gastrodia elata-notoginseng decoction, Yin-deficiency, Yang-hyperactivity syndrome, Hypertension, Inflammatory reaction, Blood pressure variability.

\section{Introduction}

As a common disease, hypertension is one of the cardiovascular diseases with the highest incidence in the world. Increased systolic pressure, high pulse pressure and blood pressure circadian dysrhythmias often occur in old people due to their atherosclerosis, increased stiffness, decreased elasticity of vessels, and hypofunction of blood pressure regulation center. In antihypertensive therapy, improvement of systolic pressure is as important as reduction of blood pressure, pressure variation and adverse events like target-organ damage $[1,2]$. Traditional Chinese medicine (TCM) therapy for hypertension has several advantages in syndrome differentiation treatment. The whole regulation, smooth pressure reduction, symptom improvement, target-organ protection, better combination with Western medicine, and attenuation synergia [3]. Gastrodia elata-notoginseng decoction contains sanchi flower, Gastrodia elata and Dendrobium officinale, which has the effect of nourishing liver and kidney as well as reducing blood pressure. Previous studies have shown that Gastrodia elata-notoginseng decoction can improve hypertensive patients with anxiety [4]. Here, we initially explored its mechanism of action through further observing intervention effect of Gastrodia elata-notoginseng decoction on old hypertensive patients with yin-deficiency and yang-hyperactivity syndrome, and monitoring changes in blood pressure variability and plasma vasoactive substance during treatment. 


\section{Materials and Methods}

Diagnostic criteria of elderly essential hypertension was referred to the Guidelines for Prevention and Treatment of Hypertension in China (1999 trial): age $\geq 65$, SBD $\geq 140$ $\mathrm{mmHg}(1 \mathrm{mmHg}=0.133 \mathrm{kPa})$ and/or DBP $\geq 90 \mathrm{mmHg}$ [2]. If $\mathrm{SBD} \geq 140 \mathrm{mmHg}$ and $\mathrm{DBP}<90 \mathrm{mmHg}$, it is defined as Isolated Systolic Hypertension (ISH).

TCM differentiation criteria were referred to Good Clinical Practice of new TCM [5]. Primary symptoms: dizziness, headache, flushing, soreness and weakness of waist and knees. Minor symptoms: palpitation, palpitation, tinnitus, bitter taste, dry throax, amnesia, red tongue with little coating and fine pulse. With 3 primary symptoms or 2 minor symptoms above, patients can be diagnosed in assistance of tongue pulse.

\section{Enrolment criteria}

(1) Meeting diagnostic criteria of elderly essential hypertension; (2) patients with yin-deficiency and yanghyperactivity syndrome; (3) age 65 80, all genders; (4) informed consent.

\section{Exclusion criteria}

(1) Various secondary hypertensive patients with severe cardiovascular and cerebrovascular diseases; (2) psychopaths or patients with disturbance of intelligence who are unable to cooperate with observers; (3) patients with myocardial infarction, stroke and cardiac failure; (4) patients with severe allergic constitution, or patients allergic to known ingredients.

\section{General data}

80 elderly essential hypertensive patients treated in TCM department and cardiology department of Jining City Hospital of TCM from 2014 to 2016 were selected and randomly divided into control group (40 cases, 22 males and 18 females) and treatment group (40 cases, 21 males and 19 females). In control group, the mean age was $69.4 \pm 5.8$; Body Mass Index (BMI) was $24.68 \pm 4.01 \mathrm{~kg} \bullet \mathrm{m}^{-2}$; mean duration was $11.8 \pm$ $10.6 \mathrm{y}$; TCM symptom aggregate score was $25.68 \pm 7.55$ points. The hypertension grade of control group was divided into Grade $1(\mathrm{n}=11)$, Grade $2(\mathrm{n}=19)$ and Grade $3(\mathrm{n}=10)$. In treatment group, the mean age was $69.8 \pm 5.9$; body mass index (BMI) was $24.56 \pm 3.97 \mathrm{~kg} \bullet \mathrm{m}^{-2}$; mean duration was 12.4 $\pm 11.6 \mathrm{y}$; TCM symptom aggregate score was $24.79 \pm 7.65$ points; the hypertension grade of treatment group was divided into Grade $1(\mathrm{n}=10)$, Grade $2(\mathrm{n}=19)$ and Grade $3(\mathrm{n}=11)$. There was no statistical significance of general data between groups $(\mathrm{P}>0.05)$.

\section{Therapeutic method}

Two groups received the same antihypertensive treatment (Lamlodipine $2.5 \mathrm{mg}$ q.d., one tablet daily), and the treatment group additionally received Gastrodia elata-notoginseng decoction (sanchi flower $6 \mathrm{~g}$, Gastrodia elata $10 \mathrm{~g}$,
Dendrobium officinale $6 \mathrm{~g}$ ). Both groups were treated for 12 weeks continuously.

\section{Observational index}

(1) $24 \mathrm{~h}$ ambulatory blood pressure monitoring (24 hABPM): dynamic electrocardiogram and blood pressure monitor CB-2301-A was used with special procedure setting (recording every $30 \mathrm{~min}$ during 8:00-22:00 and every $60 \mathrm{~min}$ during 22:00-next 8:00). Numbers of effective readings counted for over $80 \%$ of monitoring times. BPV indexes included several standard deviations, such as $24 \mathrm{~h}$ systolic pressure standard deviation (24 h SSD), $24 \mathrm{~h}$ diastolic pressure standard deviation (24 h DSD), diurnal systolic pressure standard deviation (dSSD), diurnal diastolic pressure standard deviation (dDSD), nocturnal systolic pressure standard deviation (nSSD) and nocturnal diastolic pressure standard deviation (nDSD); (2) vascular endothelial function and inflammation index examining included levels of adiponectin, resistin and CRP. Double-antibody sandwich ABC-ELISA assay was adopted in testing adiponectin and resistin, while radioimmunoassay in testing CRP. Patients were tested before and after treatment by kits (Shanghai Senxiongbiotech industrial limited company, batch number 20150961).

Evaluation of therapeutically criterion in TCM syndrome. Significant: obvious improvement in signs and symptoms, therapeutic index $\geq 70 \%$;

\section{Effective}

Improvement in signs and symptoms, $30 \% \leq$ therapeutic index $\leq 70 \%$;

\section{Invalid}

Little improvement in signs and symptoms, therapeutic index $<30 \%$;

Therapeutic index $(n)=($ score of prior treatment-score of post treatment)/score of prior treatment $\times 100 \%$

\section{Statistical approach}

Statistical software SPSS 17.0 was used for data processing. ( $\bar{x}$ $\pm s$ ) was adopted in measurement data. Independent-samples ttest was used in comparison among groups. Pairing t-test was used in intra-group comparison and chi-square test was used in enumeration data. It will be considered as statistical significance when $\mathrm{P}<0.05$.

\section{Results}

\section{Efficacy comparison of TCM syndrome between groups}

Total effective rate of TCM syndrome in treatment group was $85.0 \%$, which was higher than that of control group $62.5 \%$ $(\mathrm{P}<0.05$, Table 1). 
Effect of Gastrodia elata-notoginseng decoction on blood pressure variability and inflammatory factors in old hypertensive patients with yin-deficiency and yang-hyperactivity syndrome

\section{Comparison of mean ambulatory blood pressure between groups}

Compared with prior treatment, post-treatment $24 \mathrm{~h} \mathrm{SBP}$ and $24 \mathrm{~h}$ DBP decreased significantly $(\mathrm{P}<0.01)$. Meanwhile, $24 \mathrm{~h}$ SBP of treatment group was lower than that of control group $(\mathrm{P}<0.01$, Table 2).

Table 1. Efficacy comparison of TCM syndrome in patients (n (\%)).

\begin{tabular}{llllll}
\hline Group & Cases & $\begin{array}{l}\text { Significan } \\
\mathbf{t}\end{array}$ & Effective & Invalid & $\begin{array}{l}\text { Total } \\
\text { effective } \\
\text { rate }\end{array}$ \\
\hline Treatment & 40 & $24(60.0)$ & $10(25.0)$ & $6(15.0)$ & $34(85.0)$ \\
\hline Control & 40 & $18(45.0)$ & $11(27.5)$ & $11(27.5)$ & $30(62.5)$ \\
\hline
\end{tabular}

Note: Compared with control group, $\mathrm{P}<0.05$

Table 2. Comparison of mean blood pressure of TCM syndrome in prior-treatment and post-treatment patients $(\mathrm{mmHg}, \bar{x} \pm s)$.

\begin{tabular}{lllll}
\hline Index & Group & Cases & Prior treatment & Post treatment \\
\hline 24 h SBP & Control & 40 & $145.34 \pm 7.15$ & $130.12 \pm 3.85^{* *}$ \\
\cline { 2 - 5 } & Treatment & 40 & $145.12 \pm 6.76$ & $123.54 \pm 4.36^{* *}$ \\
\hline 24 h DBP & Control & 40 & $78.71 \pm 9.55$ & $71.59 \pm 7.64^{* *}$ \\
\cline { 2 - 5 } & Treatment & 40 & $80.42 \pm 8.52$ & $72.64 \pm 6.31^{* *}$ \\
\hline
\end{tabular}

Note: ${ }^{* *}$ Compared with prior-treatment group, $\mathrm{P}<0.01$; Compared with posttreatment control group, $\mathrm{P}<0.05$.

\section{Comparison of blood pressure variability in prior- treatment and post-treatment patients}

Compared with prior treatment, the value of post-treatment 24 $\mathrm{h}$ SSD, $24 \mathrm{~h}$ DSD, dSSD and nSSD decreased significantly $(\mathrm{P}<0.01)$. Meanwhile, $24 \mathrm{~h}$ SBP-SD, DSBP-SD and NSBP-SD of treatment group was significantly lower than that of control group $(\mathrm{P}<0.01$, Table 3$)$.

Table 3. Comparison of blood pressure variability inprior-treatment and post-treatment patients ( $\mathrm{mmHg}, \bar{x} \pm s$ ).

\begin{tabular}{lllll}
\hline Index & Group & Cases & Prior treatment & Post treatment \\
\hline 24h SBP-SD & Control & 32 & $14.63 \pm 3.65$ & $12.19 \pm 3.21^{* *}$ \\
\cline { 2 - 5 } & Treatment & 32 & $16.73 \pm 7.90$ & $10.95 \pm 2.35^{* *}$ \\
\hline 24h DBP-SD & Control & 32 & $9.82 \pm 2.67$ & $8.15 \pm 2.45^{*}$ \\
\cline { 2 - 5 } & Treatment & 32 & $10.63 \pm 5.11$ & $8.45 \pm 1.76^{*}$ \\
\hline DSBP-SD & Control & 32 & $13.81 \pm 4.04$ & $11.16 \pm 2.26^{* *}$ \\
\cline { 2 - 5 } & Treatment & 32 & $16.06 \pm 4.14$ & $10.08 \pm 2.67^{* *}$ \\
\hline DDBP-SD & Control & 32 & $9.27 \pm 2.77$ & $8.78 \pm 2.83$ \\
\cline { 2 - 5 } & Treatment & 32 & $9.21 \pm 3.16$ & $8.42 \pm 2.78$ \\
\hline NSBP-SD & Control & 32 & $12.47 \pm 4.23$ & $10.49 \pm 3.76^{*}$ \\
\cline { 2 - 5 } & Treatment & 32 & $13.65 \pm 4.92$ & $9.16 \pm 3.77^{* *}$ \\
\hline
\end{tabular}

\begin{tabular}{lllll}
\hline NDBP-SD & Control & 32 & $9.57 \pm 4.08$ & $9.36 \pm 3.98$ \\
\cline { 2 - 5 } & Treatment & 32 & $9.39 \pm 3.82$ & $8.96 \pm 3.61$ \\
\hline
\end{tabular}

Note: Compared with prior-treatment group, ${ }^{*} \mathrm{P}<0.05$ and ${ }^{* *} \mathrm{P}<0.01$; compared with post-treatment control group, $\mathrm{P}<0.05$.

\section{Levels of adiponectin, resistin and CRP in patients}

Compared with prior treatment, post-treatment levels of resistin and CRP significantly decreased while level of adiponectin increased $(\mathrm{P}<0.05)$. Besides, changes of adiponectin, resistin and $\mathrm{CRP}$ in treatment group was significantly larger than that of control group $(\mathrm{P}<0.05$, Table $4)$.

Table 4. Levels of adiponectin, resistin and CRP in patients $(\bar{x} \pm s)$

\begin{tabular}{lllll}
\hline Index & Group & Cases & Prior treatment & Post treatment \\
\hline 24 h SBP-SD & Control & 32 & $14.63 \pm 3.65$ & $12.19 \pm 3.21^{* *}$ \\
\cline { 2 - 5 } & Treatment & 32 & $16.73 \pm 7.90$ & $10.95 \pm 2.35^{* *}$ \\
\hline 24 h DBP-SD & Control & 32 & $9.82 \pm 2.67$ & $8.15 \pm 2.45^{*}$ \\
\cline { 2 - 5 } & Treatment & 32 & $10.63 \pm 5.11$ & $8.45 \pm 1.76^{*}$ \\
\hline DSBP-SD & Control & 32 & $13.81 \pm 4.04$ & $11.16 \pm 2.26^{* *}$ \\
\cline { 2 - 5 } & Treatment & 32 & $16.06 \pm 4.14$ & $10.08 \pm 2.67^{* *}$ \\
\hline NSBP-SD & Control & 32 & $9.27 \pm 2.77$ & $8.78 \pm 2.83$ \\
\cline { 2 - 5 } & Treatment & 32 & $9.21 \pm 3.16$ & $8.42 \pm 2.78$ \\
\cline { 2 - 5 } & Treatment & 32 & $13.65 \pm 4.92$ & $9.16 \pm 3.77^{* *}$ \\
\hline NDBP-SD & Control & 32 & $9.57 \pm 4.08$ & $9.36 \pm 3.98$ \\
\cline { 2 - 5 } & Treatment & 32 & $9.39 \pm 3.82$ & $8.96 \pm 3.61$ \\
\hline
\end{tabular}

\section{Discussion}

Hypertension belongs to ranges of headache and giddiness in traditional Chinese medicine, and yin-deficiency and yanghyperactivity syndrome is most common in syndrome distribution [6,7]. Previous study showed that yin-deficiency and yang-hyperactivity syndrome was related to BPV in old hypertensive patients. Internal imbalance of yin and yang as well as disturbance of $\mathrm{q}_{\mathrm{i}}$ activity often leads to hypertension, while asthenia in origin and asthenia in superficiality is the basic pathogenies. Liver and kidney are the most important parts in action mechanism where liver are substantial yin and functional yang. With kidney failing to nourish liver (Yindeficiency-type kidney), liver is influenced, which leads to hyperactivity of liver-yang $[8,9]$. Therefore, clinical therapy focuses on calming the liver, supplementing yin and regulating blood-pressure.

Gastrodia elata-notoginseng decoction in this study contains Gastrodia elata, sanchi flower, Gastrodia elata and Dendrobium officinale. With natured and sweet feature, Gastrodia elata acts as the sovereign drug that has effects of relieving convulsion and spasm, calming liver and suppressing 
yang without impairing yin. With cool-natured and sweet feature, sanchi flower acts as the ministerial drug that has effects of clearing heat, calming liver, reducing blood pressure, activating and harmonizing $\mathrm{Q}_{\mathrm{i}}$-blood, promoting blood circulation to remove blood stasis, regulating emotion and sleep, and enhancing blood pressure reduction. Dendrobium officinale acts as the assistant drug that has effects of nourishing stomach and yin, clearing heat, moistening lung, tonifying kidney, improving eyesight and strengthening waist.

Total effective rate of TCM syndrome in treatment group was $85.0 \%$, which was higher than that of control group $62.5 \%$. The result shows that Gastrodia elata-notoginseng decoction can significantly improve clinical symptoms in old hypertensive patients with yin-deficiency and yanghyperactivity syndrome.

BPV is defined as the change scope of blood pressure in a certain period, which is related to target-organ damage. Hypertension treatment focuses on reduction of mean blood pressure as well as blood pressure variability. In this study, 24 $\mathrm{h}$ SSD, 24h DSD, dSSD and nSSD of post treatment are significantly decreased than that of prior treatment $(\mathrm{P}<0.01)$. Based on comparison between groups, $24 \mathrm{~h}$ SBPV, dSBPV and nSBPV of treatment group are significantly lower than that of control group $(\mathrm{P}<0.01)$. In reduction of mean blood pressure, post-treatment $24 \mathrm{~h} \mathrm{SBP}$ and $24 \mathrm{~h}$ DBP of both groups are significantly decreased than that of prior treatment $(\mathrm{P}<0.01)$. The scope of systolic pressure reduction of treatment group is larger than that of control group $(\mathrm{P}<0.01)$, indicating that combined with general treatment of Western medicine, Gastrodia elata-notoginseng decoction can further reduce blood pressure and improve BPV in old hypertensive patients with yin-deficiency and yang-hyperactivity syndrome.

Although the etiology and pathogenesis of hypertension is unclear, previous study has shown that cytokines play an important role in pathological process of hypertension [10]. As an important factor of vascular inflammation and endothelial injury, CRP has vital function to the direct development of hypertension, CRP is considered as an independent risk factor of hypertension [11]. Besides, resistin is also recognized as one cytokines in inflammatory response, which can affect regulation of endothelial function, induction of endothelial activation and expression of multiple cytokines [12]. These proinflammatory factors destruct vessel structure and function, finally leading to endothelium dysfunction. Adiponectin always acts as one cytokines with protective effect [13]. Beside anti-atherosclerosis and anti-inflammatory effect, it can regulate CRP expression in adipose tissue and adjust CRP level. Our study shows that post-treatment CRP and resistin levels are significantly decreased while adiponectin level is significantly increased, which is more obvious in treatment group. The result indicates that Gastrodia elata-notoginseng decoction has multiple anti-inflammatory effects on hypertensive patients such as harmful factors suppression and protective factors enhancement.

Our study shows that Gastrodia elata-notoginseng decoction has significant effect of blood pressure decrease, BPV reduction, clinical symptom improvement, CRP and resistin reduction, and adiponectin increase, which provides theoretical basis for TCM treatment in old hypertensive patients with yindeficiency and yang-hyperactivity syndrome.

\section{Acknowledgement}

Fund program: Science and Technology Development Project of Traditional Chinese Medicine in Shandong Province.

\section{References}

1. Geriatric Medicine Branch of Chinese Medical Association, Council for Hypertension Research of Chinese Medical Doctor Association. Elder characteristics of hypertension and expert advice for clinical diagnosis and treatment. Chinese J Geriatr 2014; 33: 689-701.

2. Revision Committee of Guidelines for Prevention and Treatment of Hypertension in China. Guidelines for Prevention and Treatment of Hypertension in China. Chinese J Cardiol 2011; 39: 579-616.

3. Chen $X$. Characteristics and advantages of traditional Chinese medicine inprevention and treatment of hypertension. Tianjin J Trad Chinese Med 2012; 29: 155-157.

4. Wang D, Zhang X, Song G. Effects of Tianma-Sanqi decoction on blood pressure variability of hypertensive patients with anxiety. Chinese J Behav Med Brain Sci Neurology 2011; 77: 731-737.

5. Zheng X. Clinical research principles of traditional Chinese medicine. Beijing: China Medical Science Press 2002; 74-78.

6. Gu C, Ding Y. Review of hypertension syndrome. Chin Arch Tradit Chin Med 2003; 21: 1156.

7. Zou Z, Fan Y, Zhou X. TCM syndrome of hypertension in Beijing during 1996-2005. J Trad Chinese Med 2007; 48: 437.

8. Li Z, Long W, Zhang Q. Blood pressure variability and correlation studies of hypertension. Jiangsu J Tradit Chin Med 2001; 22: 16-17.

9. Gambillara V, Montorzi G, Haziza-Pigeon C. Arterial wall response to ex vivo exposure to oscillatory shear stress. J Vasc Res 2005; 42: 535-544.

10. Teng Y, Xie R, Shi X. Effect of kidney-supplementing blood-quickening decoction on blood pressure variability and life quality in old hypertensive patients with kidney deficiency and blood stasis. Chinese J Exp Trad Med Form 2016; 22: 173-177.

11. Zhao Y, Yang S, Wang X. Relation of c-reactive protein with blood pressure and hypertension. Chinese J Dis Contr Prev 2011; 15: 101-103.

12. Pyorala $M$, Miettinen $H$, Halonen $P$. Insulin resistance syndrome predicts the risk of coronary heart disease and stroke in healthy, middle-aged men: the 22-years follow-up results of the Helsinki Policemen Study. Arterioscler Thromb Vasc Biol 2000; 20: 538-544. 
Effect of Gastrodia elata-notoginseng decoction on blood pressure variability and inflammatory factors in old hypertensive patients with yin-deficiency and yang-hyperactivity syndrome

13. Ouchi N, Kihara S, AritaY. Adipocyte-derived plasma protein, adiponectin, suppresses lipid accumulation and class A scavenger receptor expression in human monocytederived macrophages.Circulation 2001; 103: 1057-1063.

\section{*Correspondence to}

Sun Bing

Teaching-Research Office of Traditional Chinese Medicine

Jining Medical University

PR China 